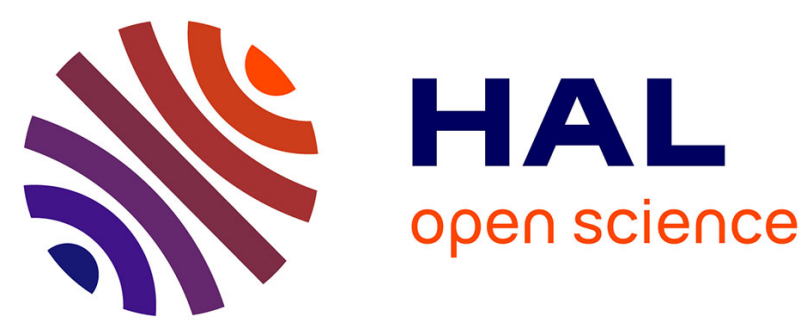

\title{
Elucidating the role of surface chemistry on cationic phosphorus dendrimer-siRNA complexation
}

Marco A. Deriu, N. Tsapis, M. Noiray, G. Grasso, Nabil El brahmi, Serge Mignani, Jean Pierre Majoral, E. Fattal, Andrea Danani

\section{- To cite this version:}

Marco A. Deriu, N. Tsapis, M. Noiray, G. Grasso, Nabil El brahmi, et al.. Elucidating the role of surface chemistry on cationic phosphorus dendrimer-siRNA complexation. Nanoscale, 2018, 10 (23), pp.10952-10962. 10.1039/C8NR01928B . hal-01957985

\section{HAL Id: hal-01957985 \\ https://hal.science/hal-01957985}

Submitted on 24 Oct 2019

HAL is a multi-disciplinary open access archive for the deposit and dissemination of scientific research documents, whether they are published or not. The documents may come from teaching and research institutions in France or abroad, or from public or private research centers.
L'archive ouverte pluridisciplinaire HAL, est destinée au dépôt et à la diffusion de documents scientifiques de niveau recherche, publiés ou non, émanant des établissements d'enseignement et de recherche français ou étrangers, des laboratoires publics ou privés. 


\section{Elucidating the Role of Surface Chemistry on Cationic Phosphorus Dendrimer-siRNA Complexation}

\section{Author names and affiliations.}

Marco A. Deriu ${ }^{1}$, Nicolas Tsapis ${ }^{2,}{ }^{*}$, Magali Noiray ${ }^{2}$, Gianvito Grasso ${ }^{1}$, Nabil El Brahmi ${ }^{3,4}$, Serge Mignani ${ }^{5}$, Jean-Pierre Majoral ${ }^{3,4}$, Elias Fattal ${ }^{2}$, and Andrea Danani ${ }^{1{ }^{1 *}}$

${ }^{1}$ Istituto Dalle Molle di studi sull'Intelligenza Artificiale (IDSIA), Scuola universitaria professionale della Svizzera italiana (SUPSI), Università della Svizzera italiana (USI), Centro Galleria 2, Manno, CH-6928, Switzerland.

${ }^{2}$ Institut Galien Paris-Sud, CNRS, Univ. Paris-Sud, Université Paris-Saclay, 92296 Châtenay-Malabry, France.

${ }^{3}$ Laboratoire de Chimie de Coordination, CNRS, 205 route de Narbonne F-31077 Toulouse Cedex 4, France

${ }^{4}$ Université de Toulouse, UPS, INPT, Toulouse, France

${ }^{5}$ Laboratoire de Chimie et de Biochimie pharmacologiques et toxicologiques, CNRS UMR 860, Université Paris Descartes, PRES Sorbonne Paris Cité, 45 rue des Saints Pères, 75006, Paris, France

\section{Corresponding authors}

*Andrea Danani, andrea.danani@idsia.ch, Scuola universitaria professionale della Svizzera italiana (SUPSI), Istituto Dalle Molle di studi sull'Intelligenza Artificiale (IDSIA), Università della Svizzera Italiana (USI), CH6928 Manno, Switzerland. - Tel +4158666 6568.

*Nicolas Tsapis, nicolas.tsapis@u-psud.fr, Institut Galien Paris-Sud, CNRS, Univ. Paris-Sud, Université ParisSaclay, 92296 Châtenay-Malabry, France. - Tel +33 146835582. 


\begin{abstract}
In the field of dendrimers targeting small interfering RNA (siRNA) delivery, dendrimer structural properties, such as the flexibility/rigidity ratio, play a crucial role on the efficiency of complexation. Nevertheless, advances in organic chemistry has allowed to develop dendrimers which differ only for a single atom on their surface terminals. This is the case of cationic phosphorus dendrimers, functionalized with either pyrrolidinium (DP) or morpholinium (DM) terminal groups, respectively. This small change was shown to strongly affect the dendrimer-siRNA complexation, leading to more efficient anti-inflammatory effects in the case of DP. Reasons of this different behavior can hardly be inferred only by biological in vitro and in vivo experiments due to the high number of variables and complexity of the investigated biological system. However, understanding how small chemical surface changes may completely modify the overall dendrimer-siRNA complexation is a significant breakthrough toward the design of efficient dendrimers for nucleic acids delivery. Here, we present experimental and computational approaches, based on Isothermal Titration Calorimetry and Molecular Dynamics simulations, to elucidate the molecular reasons behind DP and DM different efficiency and activity. Results of the present research highlight how chemical surface modifications may drive the overall dendrimer-siRNA affinity by influencing enthalpic and entropic contributions of the binding free energy. Moreover, this study also elucidates molecular reasons related to complexation stoichiometry which may be crucial in determining dendrimer complexation efficiency.
\end{abstract}

\title{
Keywords
}

cationic phosphorus dendrimers, pyrrolidinium, isothermal titration calorimetry, molecular dynamics, simulations, binding free energy, affinity, stoichiometry, siRNA, surface chemistry, dendriplex, delivery. 

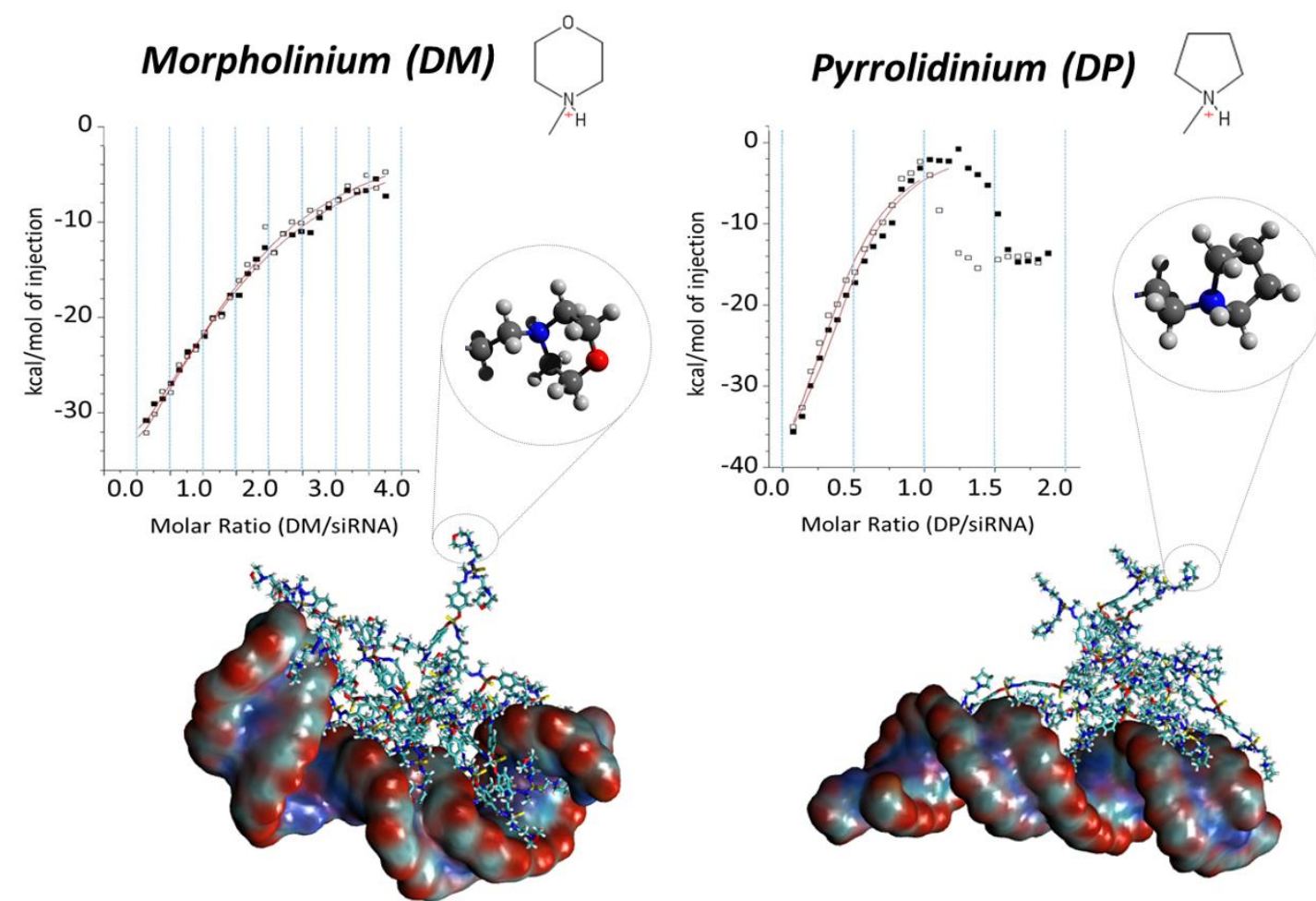

The role of surface chemistry in driving dendrimer-siRNA complexation 


\section{Introduction}

Since small interfering RNA (siRNA) administration has been proposed as a therapeutic strategy many years ago ${ }^{1}$, research has been focusing on improving siRNA penetration into cells for efficient interference ${ }^{2}$. Indeed siRNA therapy is limited by the poor penetration of naked siRNA across cell membranes due to its negative charge and large molecular weight. To efficiently cross cell membrane, siRNA are usually complexed with cationic molecules to yield positively charged complexes with a size ranging from tens to a few hundreds of nanometers. Complexation may also protect siRNA from degradation by enzymes. Complexing agents for nucleic acid delivery are numerous and include different polymeric or lipid nanocarriers such as chitosan, polyethyleneimine, cationic lipids and dendrimers. Cationic lipids or polymers complexing nucleic acids have been so far the most adapted self-assembling molecules allowing subcellular delivery of nucleic acids ${ }^{3-13}$. A promising strategy is represented by polycationic dendrimers 14,15. Dendrimers are unique tree-like branched polymers with biomolecules-like properties, low polydispersity and high degree of versatility; outstanding features for nanomedicine ${ }^{16-30}$. Dendrimer customization consists of tuning either the structure flexibility ${ }^{31-34}$ or surface properties ${ }^{35}$. Polymer flexibility has been demonstrated to play a crucial role for complexation with siRNA ${ }^{32,36,37}$. In literature, flexibility/rigidity ratio was considered a critical parameter in nanoparticle design ${ }^{38}$. For example, rigid polycationic dendrimers were shown to reorganize their peripheral groups to enhance contacts while interacting with nucleic acids ${ }^{31,32}$.

In connection with siRNA delivery, cationic phosphorus dendrimers have been demonstrated to be good candidates for gene delivery carriers after in vivo experiments ${ }^{35,39}$. In particular, in a recent study ${ }^{35}$, two different cationic, phosphorus dendrimers of generation 3 were synthesized and functionalized with either cationic pyrrolidinium or morpholinium surface groups and called DP and DM, respectively. It is worth mentioning that DP and DM differ only for their terminal groups. Both dendrimers were efficient to complex an anti TNF- $\alpha$ siRNA but complexes were shown more stable in vitro for pyrrolidinium, leading to more effective anti-inflammatory effect by inhibition of TNF- $\alpha$ both in vitro and in vivo ${ }^{35}$. These findings show that a small chemical modification may result in a significant change of the biological activity of the compounds. A complex relationship between chemical formula, structural reorganization and dendrimersiRNA binding mode might be the cause of such a significant difference. Noteworthy, this relationship can hardly be inferred only by biological in vitro and in vivo experiments due to the high number of variables and complexity of the investigated biological system. In this context, a coupled approach based on experimental and computational modelling may provide fruitful information on molecular mechanisms driving interactions of ligand-target systems ${ }^{40-42}$, such as a polymer-siRNA complexes ${ }^{15,33,34,41-43}$. Here, following this strategy, we used single-molecule resolution techniques such as Isothermal Titration Calorimetry (ITC) ${ }^{44-47}$ together with Molecular Dynamics simulations ${ }^{48-51}$, to shed light on the molecular reasons behind the DP and DM exhibited activity ${ }^{35}$. In more details, single-molecule level investigation of 
dendrimer-siRNA interactions was used to better understand how changes in dendrimer structure and biophysical properties affect the interaction with siRNA and lately emerges in a different biological activity, as recently shown ${ }^{35}$. Outcomes of the present research may also be used for further design of siRNA delivery agents characterized by the best compromise between complexation stability and release ability.

\section{Material and methods}

\section{Molecular Systems}

Phosphorus dendrimers of generation 3 were synthesized and characterized as described earlier ${ }^{35}$, leading to dendrimers bearing 48 terminal cationic pyrrolidinium groups (DP) or 48 morpholinium groups (DM) as shown in Figure 1. Dicer substrate asymmetric duplex siRNA directed against TNF- $\alpha$ as provided by Eurogentec (Liège, Belgium) as dried, purified and desalted duplexes. The TNF- $\alpha$ siRNA consisted of the following sequences:

Sense 5'-pguCUCAGCCUCUUCUCAUUCCUGct-3', Antisense 5'-AGCAGGAAUGAGAAGAGGCUGAGACAU-3',

where the lower case letters indicate 2'-deoxyribonucleotides, underlined capital letters represent 2'-Omethylribonucleotides 105 and $P$ is a phosphate residue (Mw 17,047 Da).

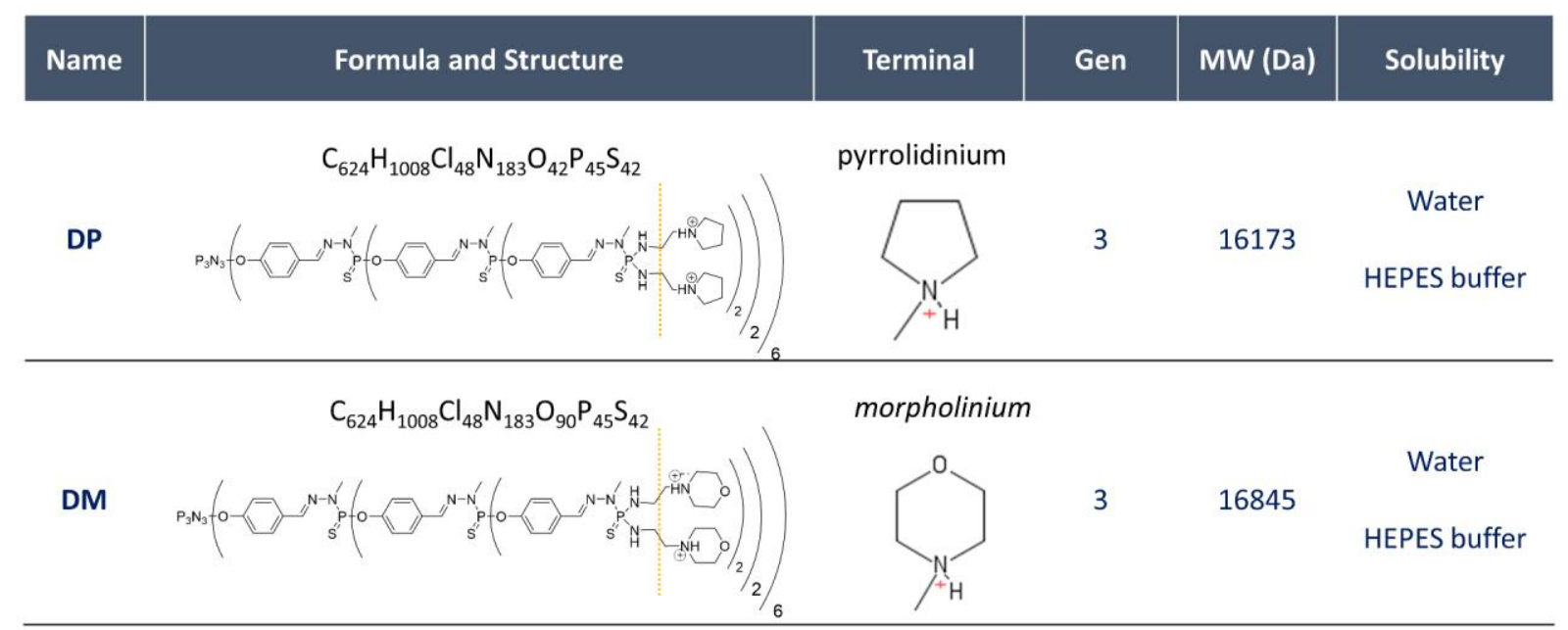

Figure 1. DP and DM Formula, Structure, Generation, molecular weight and solubility information. From a structural point of view, DP and DM differ only for the terminal group.

\section{Experimental Protocol}

A $250 \mathrm{~mL}$ of HEPES buffer was prepared at $0.01 \mathrm{M}$ and $\mathrm{pH} 7.4$ starting from a solid powder of pure HEPES (Sigma-Aldrich, France, MW $238 \mathrm{~g} / \mathrm{mol}$ ) and RNase-free water (Sigma-Aldrich, France). The same HEPES buffer was used to dilute powders of DP, DM and siRNA.

Isothermal titration calorimetry (ITC) was performed on a VP-ITC from Microcal (GE Healthcare Bio- 
Sciences, Milwaukee, WI, USA) with an active cell volume of $1.4 \mathrm{ml}$. For all experiments the dendrimer solution was injected into the siRNA solution at $10 \mathrm{~min}$ intervals, to allow equilibration between injections. The siRNA solution at $0.04 \mathrm{mM}$ was placed in the calorimetric cell and the dendrimer solution (concentration of $0.35 \mathrm{mM}$ ) was injected via the titration syringe with volume increments of $10 \mu \mathrm{L}$. Each injection produced a heat of reaction, which was determined by integration of the heat flow tracings. This mode is capable of providing both the binding isotherm and the total binding enthalpy. In Supporting Information S.1.1, Table S1 ITC setup data are reported to set up all dendrimer-siRNA binding experiments. The heat of dilution, was determined in control experiments by injecting the corresponding dendrimer dispersion into the buffer solution (Supporting Information S.1.2). The heats of dilution were subtracted from the heats determined in the corresponding dendrimer-siRNA binding experiments. For each tested dendrimer (DM, DP), one dilution and two binding experiment repetitions were performed (Table S1). The injection heats were calculated using the Microcal, LLC ITC package for Origin ${ }^{\circledR}$ version 7.0.

\section{Simulation Protocol}

\section{System Set Up}

The 3D models of two different dendrimers were developed by using Avogadro chemical editor ${ }^{52}$ and Linux bash scripts made in house (Supporting Information S.1.3). The General Amber Force Field (GAFF) ${ }^{53}$ was considered to describe the DM and DP dendrimer topologies. Partial charges were calculated considering separate residues by the RESP fitting method at the HF/6-31G level of theory using AM1-BCC charges as in a number of previous works $34,37,47,54,55$. A proper distribution of the partial charges was provided, which correctly takes into account the conformation of dendrimer and related bonds. Then, the topology and the parametrization were developed using antechamber and GROMACS associated tools ${ }^{56-60}$ and a in house code. AMBER99SB-ILDN force-field ${ }^{61-63}$ were chosen to describe the topology of the siRNA double strand.

\section{Molecular Dynamics}

For both DM and DP systems the following procedure was followed. siRNA and dendrimer 3D models were positioned at a starting distance of about $2 \mathrm{~nm}$, in a dodecahedron box filled with explicit water molecules (TIP3P) and ions (NA+ and CL-) at a physiological concentration (0.15 M). The resulting molecular systems (DP-siRNA and DM-siRNA) were composed by roughly 81000 interacting particles. Each system was energy minimized by 1000 steps of steepest descent energy minimization algorithm. A 100 ps position restrained MD was performed at $300 \mathrm{~K}^{64}$ and $1 \mathrm{~atm}{ }^{65}$. Finally, 5 repetitions of $100 \mathrm{~ns}$ long MD were performed in the NVT ensemble. Atom velocities were randomly initialized following a Maxwell Boltzmann distribution. In total, 500 ns of MD was performed to sample the conformational space of each considered system (DMsirNA and DP-siRNA). GROMACS 5 package was used for all MD simulations and data analysis ${ }^{60}$. Longranged electrostatic interactions were calculated at every step with the Particle-Mesh Ewald method with a cut-off of $1 \mathrm{~nm}$. A cut-off of $1 \mathrm{~nm}$ was also applied to Lennard-Jones interactions. The LINCS algorithm ${ }^{66}$ 
approach allowed an integration time step of $2 \mathrm{fs}$. The Visual Molecular Dynamics (VMD) ${ }^{67}$ package was used for the visual inspection of the simulated systems. To calculate the binding enthalpy the well-known "Molecular Mechanics / Generalized Born model augmented with the solvent accessible surface area term (GBSA)" approach has been considered, as employed in earlier literature to estimate and compare binding energies calculated from MD simulations ${ }^{43,68-73}$.

As a comparison term, a 100 ns MD was also performed for a siRNA double strand alone in water and ions. System preparation followed the same procedure described above for the dendrimer-siRNA systems.

\section{Results \& Discussion}

Dendrimer customization consists of tuning either the structure flexibility ${ }^{31-34}$ or surface properties ${ }^{35}$. Polymer flexibility has been demonstrated to play a crucial role for complexation with siRNA ${ }^{32,36,37}$. In literature, flexibility/rigidity ratio was considered a critical parameter in nanoparticle design ${ }^{38}$. For example, rigid polycationic dendrimers were shown to reorganize their peripheral groups to enhance contacts while interacting with nucleic acids ${ }^{31,32}$. Instead, this work focuses mostly on the latter strategy, where slight chemical modifications on dendrimer terminals may result in significant differences in dendrimer efficiency in binding siRNA and drive the biological activity of the dendriplexes ${ }^{35}$. In fact, DM and DP are both generation 3 dendrimers, and differ for 1 atom in the terminal groups. Hence, in this case, the relationship between dendrimer flexibility and siRNA complexation, well explained in earlier literature ${ }^{31-34}$, may not be a sufficient argumentation to shed light on reasons behind the DP improved efficacy and biological activity ${ }^{35}$. Here instead, the surface chemistry plays a pivotal role in driving phenomena at the nanoscale level, which results at higher scales in thermodynamic properties such as binding free energy, enthalpy/entropy balance and stoichiometry as shown by experimental and computational results in the following.

\section{Experimental Results}

ICT experiments allowed to characterize the interaction properties ${ }^{44,74-76}$ of the investigated systems DM/siRNA and DP/siRNA following set-up reported in (Supporting Information S.1.1, Table S1). In a greater detail, the binding curve obtained from a plot of the heats from each injection against the dendrimer-siRNA ratio is shown in Figure 2. The binding curve is analyzed with the one site binding model to determine dissociation constant $\left(k_{\mathrm{d}}\right)$, stoichiometry $(N)$, reaction enthalpy $(\Delta H)$, entropy $(\Delta S)$ and free energy $(\Delta G)$, quantified in Table 1. Interestingly, differently from DM-siRNA, the DP-siRNA system showed a deviation from the one site model beyond a molar ratio of 1 , in both experimental repetitions. This deviation might be related to supramolecular features of DP-siRNA complexes. A slight DP tendency to self-assembly was also inferred by dilution experiments (Supporting Information S.1.2). 

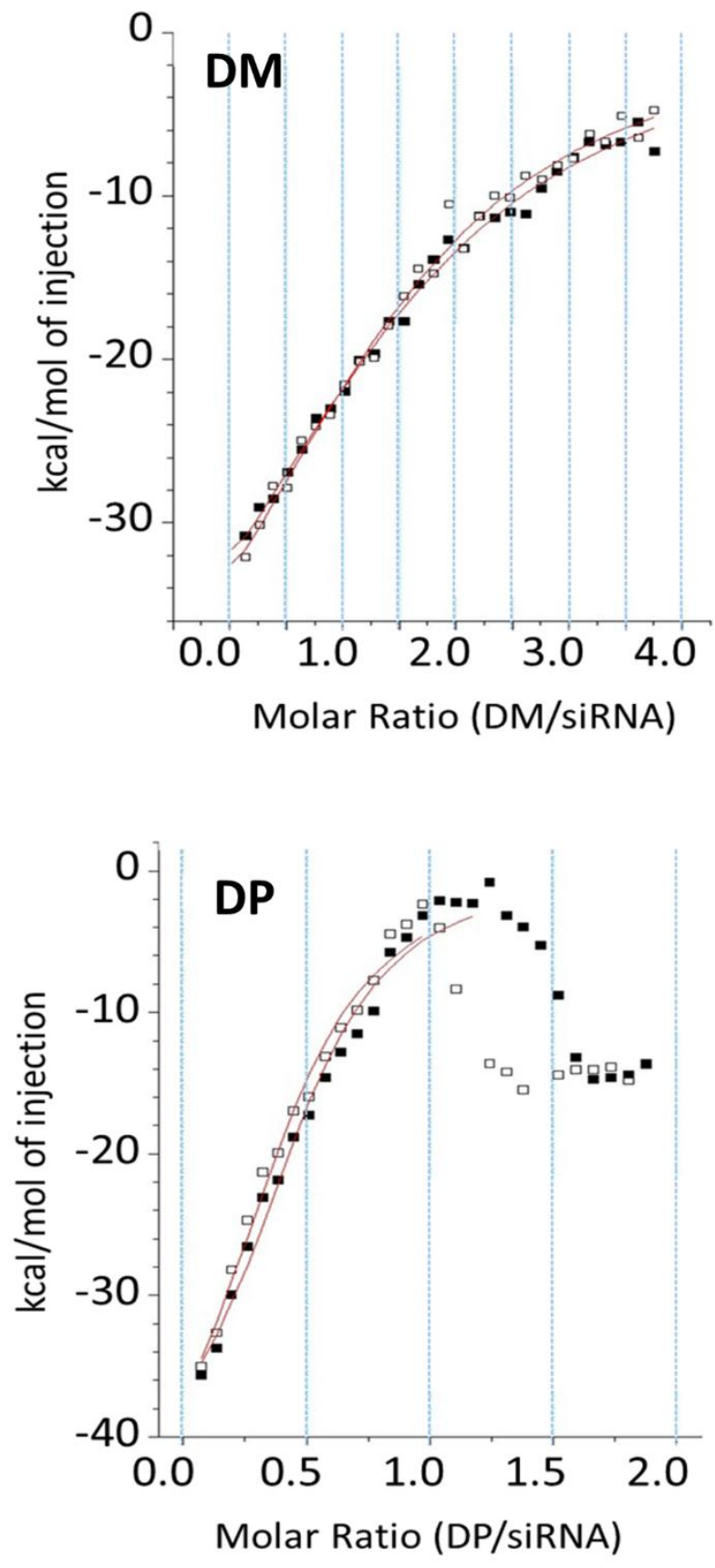

Figure 2. Two experiment repetitions (black and white squares) are reported for both DM-siRNA (top panel) and DPsiRNA (bottom panel) investigated systems. The solid red lines are the calculated curves using the best-fit parameters. The one site model was used to fit the data. Dilution signal was subtracted from titration signals before fitting.

Based on ITC data, DP showed a higher affinity with respect to DM $\left(k_{d}{ }^{D P} / k_{d}{ }^{D M}\right.$ equal to $\left.4.02 \pm 1.06\right)$. Interestingly, the enthalpic contribution is quite similar, slightly lower for DP $\left(\Delta H^{D P} / \Delta H^{D M}\right.$ equal to $0.88 \pm 0.06)$, meaning that the conformational entropy plays a role in determining the higher DP affinity. This property is exponentially related to binding free energy $\Delta \mathrm{G}=\Delta \mathrm{H}-\mathrm{T} \Delta \mathrm{S}, \Delta \mathrm{H}$ being the binding enthalpy, $\mathrm{T}$ the temperature and $\Delta S$ the contribution related to conformational entropy change from the unbound to the bound state. Interestingly, the binding free energy is higher in case of DP-siRNA complex, if compared with 
$\mathrm{DM}\left(\Delta G^{D P} / \Delta G^{D M}\right.$ equal to $\left.1.17 \pm 0.02\right)$, despite $\mathrm{DM}$ showed the highest enthalpic contribution $\left(\Delta H_{D P} / \Delta H_{D M}\right.$ equal to $0.88 \pm 0.06$ ). This means that the higher affinity of DP is likely more associated with a lower entropy reduction associated with the system complexation $\left(\triangle S^{D P} / \triangle S^{D M}\right.$ equal to $\left.0.84 \pm 0.07\right)$. In other words, after complexation, the conformational space accessible to DP-siRNA, with respect to the unbound condition, is less reduced than in the case of DM-siRNA complex. The above-mentioned differences are statistically significant considered standard deviations obtained by averaging independent experiment repetitions for both DM- and DP-siRNA complexation.

Table 1. Main results for DM-siRNA and DP-siRNA investigated systems in terms of stoichiometry $(N)$, dissociation constant $\left(k_{\mathrm{d}}\right)$ and binding enthalpy $(\Delta H)$, entropy $(\Delta S)$ and free energy $(\Delta G)$.

\begin{tabular}{clrrc}
\hline Property & \multicolumn{1}{c}{ Unit } & \multicolumn{1}{c}{ DP } & \multicolumn{1}{c}{ DM } & \multicolumn{1}{c}{ DP/DM } \\
\hline$N_{\text {siRNA/dendrimer }}$ & $\#$ & $2.25 \pm 0.03$ & $0.60 \pm 0.03$ & $3.75 \pm 0.53$ \\
$k_{d}$ & $\mu M$ & $5.60 \pm 0.85$ & $21.49 \pm 2.11$ & $4.02 \pm 1.06$ \\
$\Delta H$ & $\mathrm{kcal} / \mathrm{mol}$ & $-47.00 \pm 2.83$ & $-53.25 \pm 0.21$ & $0.88 \pm 0.06$ \\
$T \Delta S$ & $\mathrm{kcal} / \mathrm{mol}$ & $-39.65 \pm 2.95$ & $-46.96 \pm 0.21$ & $0.84 \pm 0.07$ \\
$\Delta G$ & $\mathrm{kcal} / \mathrm{mol}$ & $-7.35 \pm 0.12$ & $-6.29 \pm 0.00$ & $1.17 \pm 0.02$ \\
\hline
\end{tabular}

Another interesting feature emerging for DP can be deduced by stoichiometry data, which indicated the multivalent character of siRNA binding to DP. In detail, the estimated number of siRNA molecules bound to each DP dendrimer is roughly four times higher than DM.

Summarizing, whereas DP and DM showed a comparable binding enthalpy, and binding free energy, stoichiometric data let suppose multivalence properties for DP with roughly 2 siRNA molecules bound to a single dendrimer. By contrast, only 0.5 to one siRNA binds to DM. In agreement with recent biological experiments ${ }^{35}$, data coming from ITC (Table 1) suggested DP as more efficient toward a stable dendrimersiRNA complex. In particular DP was characterized by higher affinity with respect to DM and higher stoichiometry indicating that DP is able to bind 2 siRNA molecules, whereas DM binds less than 1 on average.

\section{Computational}

Although ITC technique was able to quantify the above mentioned properties with high precision, it is still limited in elucidating molecular phenomena and mechanisms of action, which can be inferred as hypotheses based on the experimental evidence. Computational modelling may help toward a rational description of the molecular phenomena characterizing dendrimer-siRNA interaction to shed light on cause-effect relationships ruling binding properties of dendrimer-siRNA systems. In this context, the dendrimer-siRNA interaction mechanism has been investigated by means of MD simulations as described in Material and Method section. For each dendrimer-siRNA system, data analysis has been performed taking the last $20 \mathrm{~ns}$ of each MD replica (five replicas for each system) as a statistical ensemble. 
A first observation concerns the complexation kinetics, which in both systems is very fast. A qualitative view of the complexation kinetics is given by analyzing the buried surface vs time curves for all simulations (Figure 3a, and Figure 3b). In all cases, three main phases can be identified: during the first 20 ns the dendrimer approaches the siRNA molecule and start binding to it as clearly showed by buried surface curves (Figure 3a, and Figure 3b). Then, the structures rearrange to optimize the interaction surface until a structural stability is reached, at most, in the last $20 \mathrm{~ns}$.

In average, the total interaction surface characterizing the DM-siRNA complex is significantly larger than DP-siRNA complex, as it is for both the hydrophilic and hydrophobic components (Figure 3c). This result reflects also on the binding enthalpy (Figure $3 \mathrm{~d}$ ) which is significantly higher in case of DM-siRNA complex. To calculate the binding enthalpy the well-known "Molecular Mechanics / Generalized Born model augmented with the solvent accessible surface area term (GBSA)" approach has been considered. It is worth mentioning that the ratio $\Delta H^{D P} / \Delta H^{D M}=0.74 \pm 0.13$ extracted by MM/GBSA calculations applied to DMand DP-siRNA simulations is in excellent agreement with experimental ITC data $\left(\Delta H^{D P} / \Delta H^{D M}\right.$ equal to $0.88 \pm 0.06$ reported in Table 1$)$. 
a)

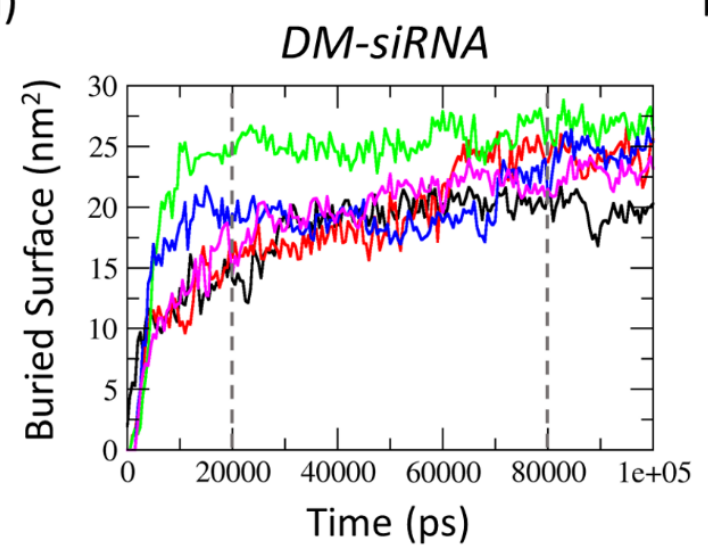

c)

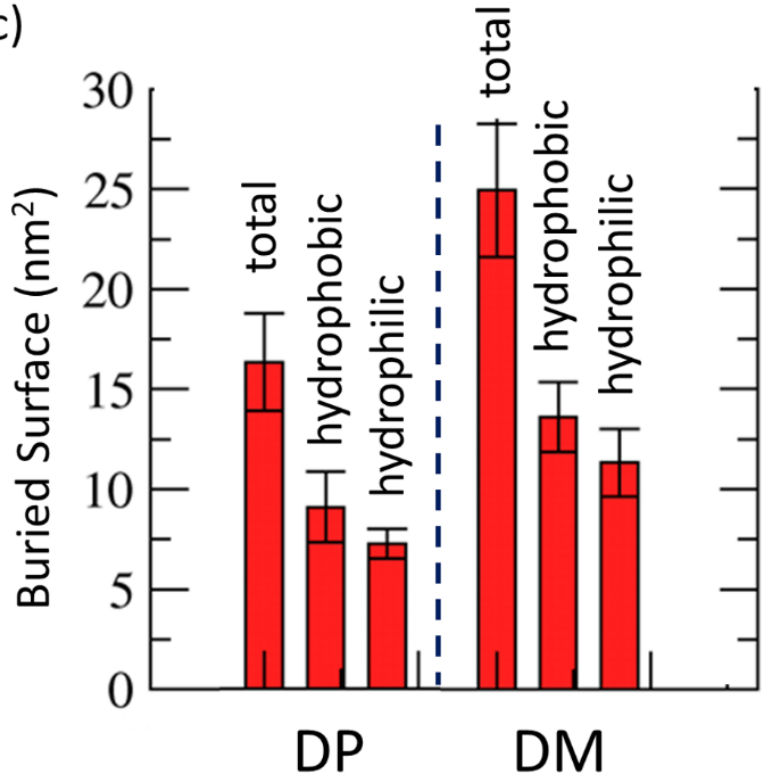

b)

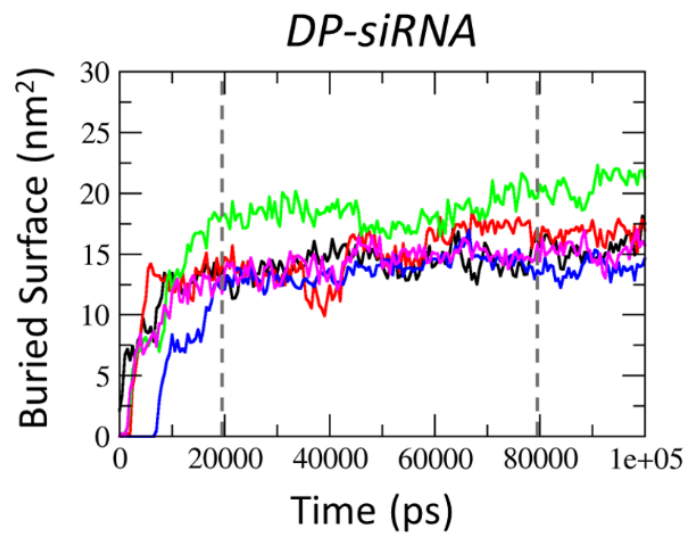

d)

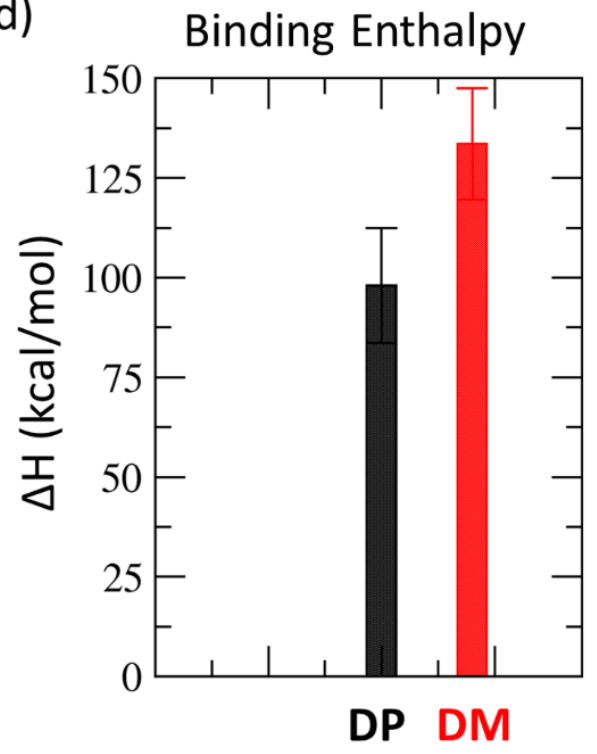

Figure 3. Dendrimer-siRNA buried total surface vs. time curves, calculated for 5 repetitions of $100 \mathrm{~ns}$ long MD simulations for DM-siRNA (a) and DP-siRNA (b). Decomposition of interaction surface in its hydrophobic and hydrophilic components (c) calculated as average \pm standard deviation over the last $20 \mathrm{~ns}$ of all replicas taken as ensemble. Binding Enthalpy was also calculated as average \pm standard deviation over the last $20 \mathrm{~ns}$ of all replicas taken as ensemble.

With the aim of highlighting the influence of the dendrimer macromolecule on the siRNA structural arrangement, representative snapshots of dendrimer-siRNA complexes are also shown in Figure 4.

MD simulations revealed that the dendrimer-siRNA complexation may drive the siRNA toward a significant conformational change as already detected in earlier literature ${ }^{77}$. In detail, the visual inspection of the MD snapshots reported in Figure 4, let suppose a strong effect of the DM in driving a siRNA conformational change. 

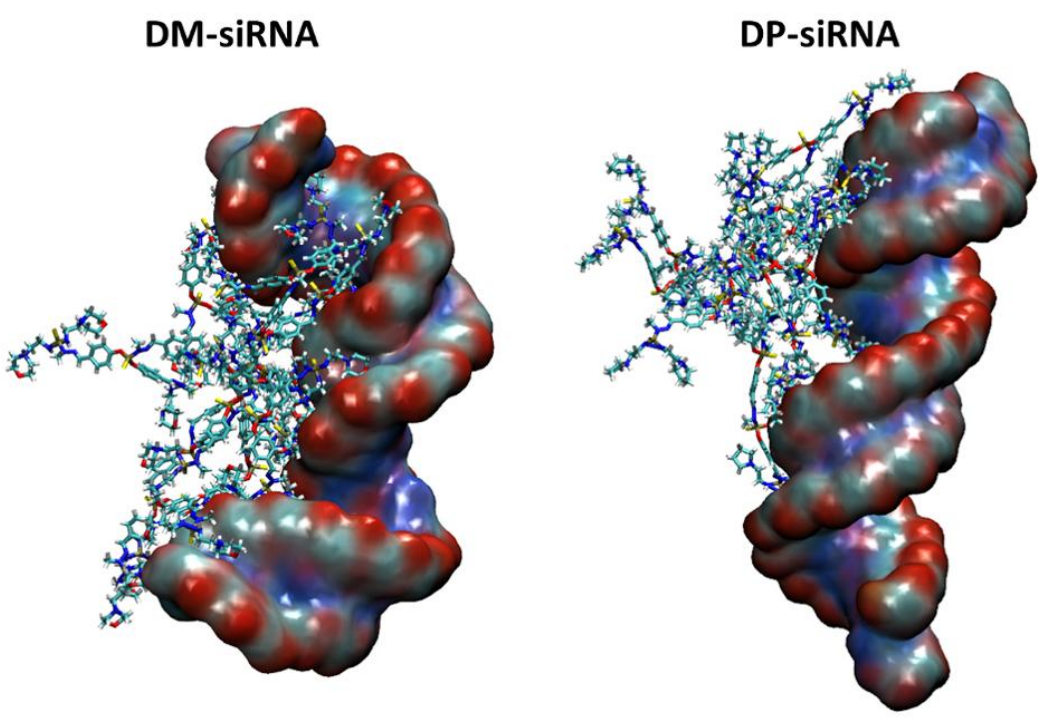

Figure 4. Representative snapshots of DM-siRNA complex (left) and DP-siRNA complex taken from the last $20 \mathrm{~ns}$ of a MD trajectory among the 5 replicas.

To provide a quantitative measure of the previously mentioned siRNA conformational modification, we have considered the angle parameter, $\vartheta$, as reported in the following. The angle $\vartheta$ was calculated as the angle between two vectors, each connecting the center of the siRNA filament to an extremity (Figure 5, right pictures). siRNA center and the extremity positions were calculated as a point identified by the center of mass (COM) of 3 selected basis pairs (Figure 5 bottom, right). Noteworthy, the DP-siRNA interaction did not induce any significant conformational changes of the siRNA macromolecule during complexation, as shown in Figure 5. One can infer that the explored angle $\vartheta$ (Figure 5, black bar) does not significantly differ from $\vartheta$ explored by a siRNA alone in water (Figure 5, green bar). A significant difference was observed in case of DM, where a larger conformational change was detected due to the siRNA molecule wrapping around the dendrimer. This molecular event was quantified by a high reduction of the $\vartheta$ parameter characterizing siRNA curvature, i.e., $\theta=120 \pm 45^{\circ}$ (Figure 5, red bar). Noteworthy, $\vartheta$ average and standard deviation are calculated by all the conformations sampled in the last $20 \mathrm{~ns}$ of the $5 \mathrm{MD}$ replicas, to increase the statistical significance of the analysis. Moreover, this result is in line with the calculated buried surface in case of DM-siRNA, much higher than the interaction surface characterizing the DP-siRNA complex. 

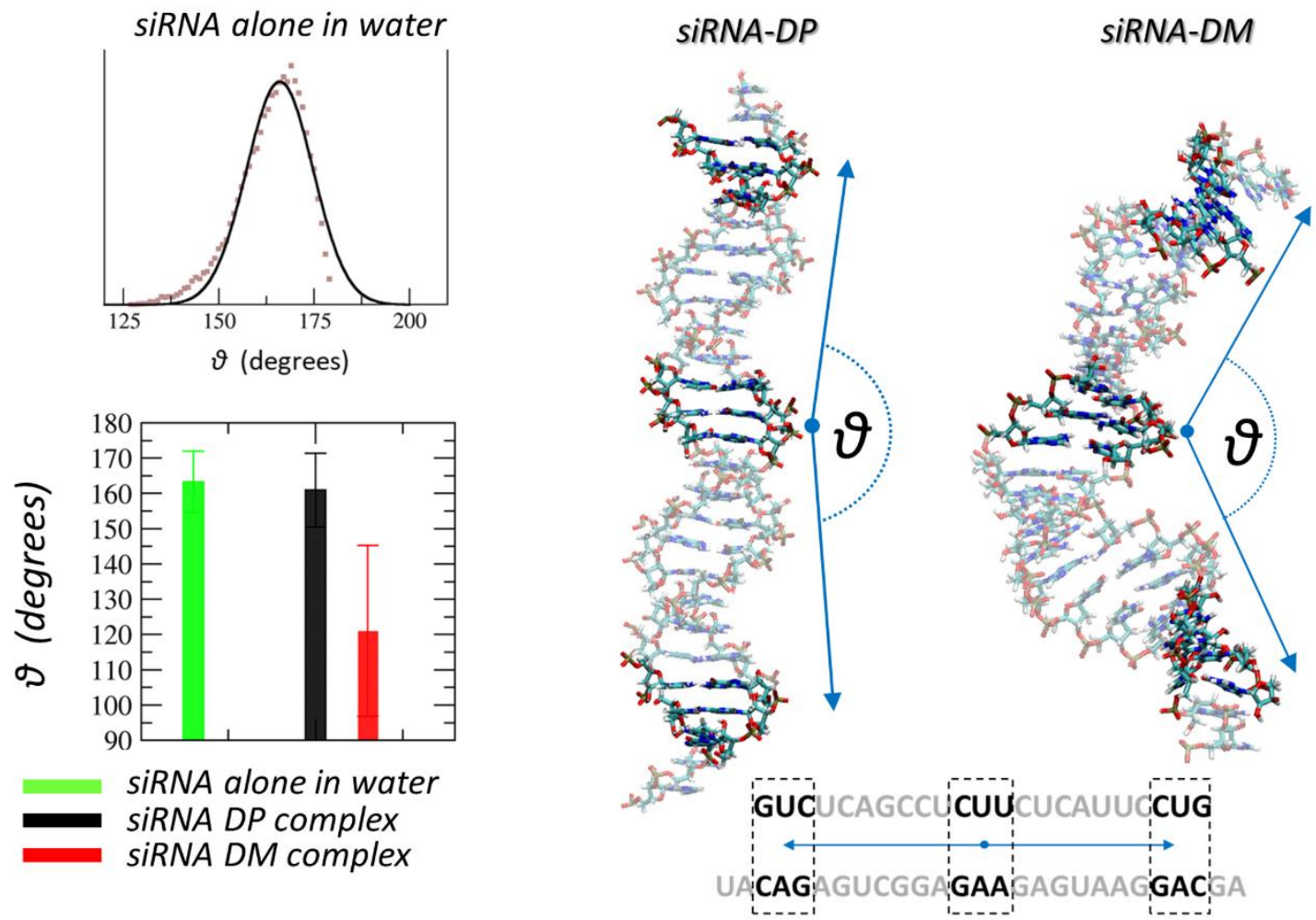

Figure 5. Effect on siRNA structural conformation due to interaction with the dendrimer (dendrimer is not shown in the picture). The angle $\theta$ is calculated between two vectors connecting the center of the filament to extremity bases along the filament axis. Center of mass of siRNA center and extremity segment are calculated by selecting 3 basis pairs (selected bases are shown in the figure on the siRNA sequence). The angle value is calculated step by step throughout the overall equilibrium MD. In case of siRNA alone in water $\theta$ is quantified as $163 \pm 9^{\circ}$ (green bar). Average and standard deviation are obtained by fitting with a gaussian distribution the simulation data (top-left panel) in the last 50 ns. Interestingly the DM-siRNA complex is characterized by a nucleic acid structure significantly bent (red bar, $\theta=120 \pm 25^{\circ}$ ), whereas the DP does not seems to affect the average conformation of siRNA (bottom left panel, black bar). For dendrimer-siRNA complexes, angle average and standard deviation are calculated by all the conformations sampled in the last $20 \mathrm{~ns}$ of the $5 \mathrm{MD}$ replicas.

A precise information on molecular phenomena driving the dendrimer-siRNA complexation is given by the Radial Distribution Function (RDF) as a function of dendrimer-siRNA distance (Figure 6). RDFs have been calculated for two well defined atom groups in the dendrimer terminals (namely T1 and T2) and siRNA (namely, groove and backbone). T1 group is composed by protonated nitrogen atoms, present in both morpholinium (DM) and pyrrolidinium (DP) terminals. T2 group is composed by the oxygen atoms in morpholinium terminal and, for the sake of comparison, one carbon atom in the pyrrolidinium terminal ring (T1 and T2 are clearly shown in Figure 6). Figure 6 shows three main curves for DM-siRNA and DP-siRNA complexes. The blue curves in Figure 6 show the RDF of T1 group with respect to siRNA groove, as constituted by electronegative $\mathrm{N}$ atoms on $\mathrm{A}, \mathrm{U}, \mathrm{C}$, and $\mathrm{G}$ bases. The red curves in Figure 6 show RDF of T2 group with respect to siRNA groove as constituted by positively charged $\mathrm{NH}$ on $\mathrm{A}, \mathrm{U}, \mathrm{C}$, G bases. Finally, the 
black curves in Figure 6 shows the RDF of T1 and T2 taken together with respect to siRNA backbone identified by ribose $P$ atoms.

Interestingly, the main difference between DM and DP lies on T2 group (Figure 6, red curve) which, in case of DM-siRNA complex, are characterized by a peak at a distance $0.2 \mathrm{~nm}$ (primary interactions with siRNA groove), indicating that DM terminals are completely embedded in the siRNA double strand. As expected, other primary interaction peaks (distance peak $0.35 \mathrm{~nm}$ ) were found in both complexes (DM-siRNA and DPsiRNA) in T1-siRNA groove RDF (Figure 6, blue curve) and T1T2-siRNA backbone (Figure 6, black curve) RDF.

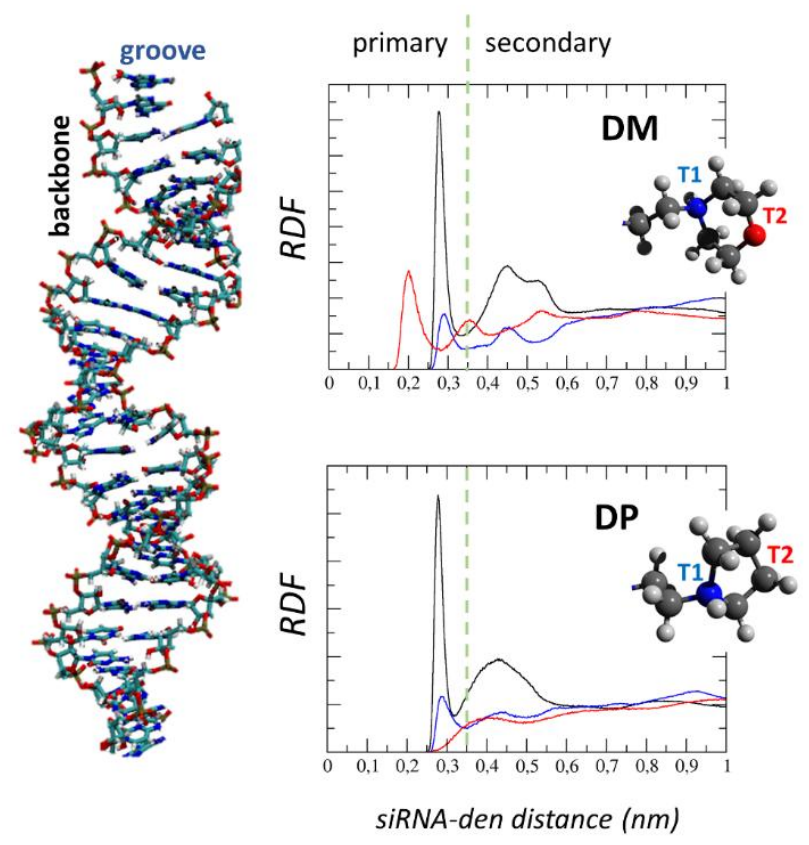

Figure 6. Radial Distribution Function (RDF). The distribution of atom-atom distances have been calculated between: atom T1 and siRNA groove, electronegative $\mathrm{N}$ on A,U,C,G bases (blue curve); atom T2 and siRNA groove, i.e., positive NH on A,U,C,G bases (red curve); atoms T1 and T2 and siRNA backbone, constituted by atoms P and bound oxygens (black curve). T1 is the charged Nitrogen, present in both pyrrolidinium and morpholinium dendrimer terminal type. T2 is instead the oxygen atom in the morpholinium terminal of DM. For comparison reasons a carbon atom has been chosen to represent T2 in the pyrrolidinium terminal of DP. RDFs have been calculated on the statistical ensemble constituted by frames in the last $20 \mathrm{~ns}$ of all $5 \mathrm{MD}$ replicas for each dendrimer-siRNA complex.

Figure 4 and Figure 6 suggest the key role played by dendrimer terminals in the dendrimer-siRNA complexation. In greater detail, although DP and DM differ only for one atom, this change influences the balance between hydrophilic and hydrophobic character of the dendrimer. The morpholinium oxygen bears a partial negative charge which makes the morpholinium terminal slightly more polar than the pyrrolidinium terminal. Interestingly, the above-mentioned difference on terminals does not result in any significant change in terms of dendrimer structural properties when the dendrimer is considered alone in water. Instead, the higher DM terminals' polarity plays a crucial role when the dendrimer complexes with siRNA as clearly highlighted by RDF curves shown in Figure 6 and by the higher DM-siRNA interaction surface (Figure 3c). 
Taken together MD results shown in Figure 3, Figure 4, Figure 5, and Figure 6 are in excellent agreement with experimental data. More in detail, MD simulations showed a DM higher binding enthalpy and buried surface (Figure 3c, and d). The molecular explanation of this DM-siRNA stronger interaction may be ascribed to the presence of morpholinium oxygens which showed a higher tendency to non-covalently bind to siRNA groove (RDF red curve in Figure 6). On the other hand, this DM molecular characteristic is paid on the entropic side with a reduced multiplicity of the accessible conformational microstates of the dendriplex. In a greater detail, the siRNA molecule is constrained to assume a curved and wrapped arrangement around DM, being anchored by morpholinium oxygens (Figure 4, and Figure 5). Differently, DP employs only protonated $\mathrm{N}$ atoms on pyrrolidinium terminals to interact with the siRNA groove and does not affect the siRNA conformation when complexed with it. In other words, DP showed a higher plasticity in complexing siRNA.

In summa, experimental and computational evidences highlighted the higher complexation stability of the DP-siRNA dendriplex. More in detail, the complexation stability is here quantified by the complexation free energy, sum of two contributions: i) the enthalpic contribution, which depends on the interaction strength (the deeper is the energy minimum, the more stable is the complex); ii) the entropic contribution, which depends on the ability of the bound system (e.g., dendriplex) to explore the conformational space with respect to the unbound condition (non-interacting dendrimer|siRNA systems). The more constrained is the dendriplex, the lower is the complexation stability.

DP and DM behave similarly when alone in water. Hence, the conformational entropy of non-interacting DM|siRNA and DP|siRNA unbound systems can be reasonably supposed comparable. The DM-siRNA bound state is characterized by a stronger interaction strength $\left(\Delta H^{D P} / \Delta H^{D M}\right.$ equal to $0.88 \pm 0.06$ reported in Table 1). However, the dendriplex is limited in exploring the conformational space (high DM-siRNA interaction surface and siRNA wrapped around the dendrimer). Instead, the DP-siRNA complex is characterized by a lower interaction strength, but the DP plasticity allows for a larger exploration of the dendriplex conformational space. In practice, DP can bind the siRNA in many more different ways with respect to DM, thus making the DP-siRNA bound state more likely.

Hence, the higher stability of the DP-siRNA complex results by a clever combination of enthalpic and entropic contribution to the complexation free energy.

In addition to previous evidences, the terminal-siRNA distance analysis, in Table 2, provides fruitful information on dendrimer terminals' contribution to dendrimer-siRNA interaction. In a greater detail, the percentage of dendrimer terminals found at selected distance range from the siRNA is reported (Table 2). In Table 2 only average values are reported, calculated by considering as an ensemble the trajectory snapshots in last 20 ns of all five replicas per each investigated complex. Standard deviations are not reported since always one order of magnitude lower than the related average value. 
Four main distance ranges were defined in Table 2: "Primary Interactions $(\mathrm{d} \leq 0.35 \mathrm{~nm})$ " range indicates terminals mostly involved in stabilizing the dendrimer-siRNA complexation; "Secondary Interactions (0.35 > $\mathrm{d} \leq 0.45 \mathrm{~nm}$ )" range indicates terminals which still contribute to dendrimer-siRNA complexation; "Close to siRNA ( $d>0.45 \mathrm{~nm} \leq 1 \mathrm{~nm}$ )" range indicates terminals which can be recruited to enhance or stabilize the complex; "Free terminals $(\mathrm{d}>1 \mathrm{~nm})$ " range indicates terminals considered free to move under thermal fluctuations and not involved into interactions. In fact, the short-ranged cut-off was set-up to $1 \mathrm{~nm}$, meaning that the van der Waals interactions are not even calculated beyond this distance.

DM employs the $57 \%$ of terminals (52.11\% of primary interactions $+4.59 \%$ of secondary interactions) to optimize the complexation with siRNA, leaving the $25 \%$ of its terminals free and eventually available to catch another siRNA molecule. This is in line with a higher buried surface and also a higher binding enthalpy with respect to DP. It is also interesting to notice (Figure 4) how DM attaches both the siRNA center and the extremities exerting a bending action on the siRNA as quantified by the angle parameter $\vartheta$ described above (Figure 5). On the other hand, DP behaves in an opposite way. DP employs the $35 \%$ terminals (31.97\% of primary interactions $+3.26 \%$ of secondary interactions) to optimize the complexation with siRNA, leaving the $49 \%$ of its terminals free to move. This implies a higher ability of DP to bind other siRNA molecules.

Table 2. Terminal-siRNA distance. The percentage of dendrimer terminals found at a selected distance range from the siRNA is reported. Four main distance ranges are defined: "Primary Interactions $(\mathrm{d} \leq 0.35 \mathrm{~nm})$ " range indicates terminals mostly involved in stabilizing the dendrimer-siRNA complexation; "Secondary Interactions $(0.35>\mathrm{d} \leq 0.45$ $\mathrm{nm})$ " range indicates terminals which still contribute to dendrimer-siRNA complexation; "Close to siRNA (d $>0.45 \mathrm{~nm}$ $\leq 1 \mathrm{~nm}$ )" range indicates terminals which can be recruited to enhance or stabilize the complex; "Free terminals $(\mathrm{d}>$ $1 \mathrm{~nm})$ " range indicates terminals considered free to move under thermal fluctuations and not involved into interactions. Percentage values have been calculated on statistical ensemble constituted by frames in the last 20 ns of all 5 MD replicas for each dendrimer-siRNA complex. Standard deviations are not reported since always one order of magnitude lower than the related average value.

\begin{tabular}{c|cccc} 
& $\begin{array}{c}\text { Primary Interactions } \\
0.35 \mathrm{~nm} \leq d\end{array}$ & $\begin{array}{c}\text { Secondary Interactions } \\
0.35 \mathrm{~nm}<d \leq 0.45 \mathrm{~nm}\end{array}$ & $\begin{array}{c}\text { Close to siRNA } \\
0.45 \mathrm{~nm}<d \leq 1 \mathrm{~nm}\end{array}$ & $\begin{array}{c}\text { Free Terminals } \\
d>1 \mathrm{~nm}\end{array}$ \\
\hline DM & $52.11 \%$ & $4.59 \%$ & $18.70 \%$ & $24.60 \%$ \\
DP & $31.97 \%$ & $3.26 \%$ & $15.84 \%$ & $48.93 \%$
\end{tabular}

Summarizing, DP employs only a lower percentage of its terminals to complex siRNA with respect to DM, an interesting feature which was also observed in experimental ICT stoichiometry data. In greater detail, data in Table 1 suggest DP able to bind around 2 siRNA molecules and DM less than one. In close agreement, computational data quantifying the percentage of dendrimer terminals effectively involved in the dendriplex formation (Table 2), indicated that DP employs only $35 \%$ of its terminals to provide the complexation, whereas the $49 \%$ of terminals are free to fluctuate under thermal motions and eventually to 
catch another siRNA. Instead, DM employs the most part of its terminals (57\%) to complex the siRNA and leaves free only $25 \%$.

But there is more, DM-siRNA complexes are also characterized by DM terminals anchoring and pulling the siRNA to be wrapped around the dendrimer. Hence, the complexation mechanisms involved also a significant conformational change of the siRNA. This may result in a competitive mechanism among DM particles in solution, which, on one side repel each other due to the negative charged surface (because of morpholinium oxygens not present in DP), and on the other side sequestrates siRNA and forces it to wrap around the dendrimer.

Taken together, experimental and computational results of the present research suggest how little modifications of the dendrimer surface chemistry (e.g., DP and DM differing for a single atom in the terminal group) may significantly affect the mechanism driving dendriplex complexation.

In greater detail, DP exhibits a collaborative strategy where a single DP is able to link more than one siRNA without requiring it to wrap around the dendrimer. Therefore, more than one siRNA may interact with one dendrimer, but also more than one dendrimer may attach the same siRNA in different areas. This might explain the $2.25 \pm 0.03$ stoichiometry and the self-assembly behavior that ITC binding curves with molar ration higher than 1 suggest (Figure 2). On the other hand DM-siRNA interaction consists of dendrimer and siRNA in a one-to-one exclusive interaction. A competition between DM dendrimers to bind a single siRNA might explain the $0.60 \pm 0.03$ stoichiometric value.

In conclusion, DP is more efficient than DM in binding siRNA due to the higher plasticity in the complexation dynamics. Due to the lower dendrimer-siRNA interaction strength, DP may also be more efficient than DM in releasing siRNA under specific conditions.

Depending on the specific application, one could even think to play more with surface chemistry and, for example, to design hybrids with a different percentage of pyrrolidinium and morpholinium terminals to tailor the balance between enthalpic and entropic contributions resulting in desired affinity and stoichiometry.

\section{Conclusions}

The study here proposed investigated the effect of surface chemistry on dendrimer-siRNA complexation dynamics and mechanism. More in detail, this research considered two different cationic, phosphorus dendrimers of generation 3, namely DP and DM, differing only for one atom in the terminal group. Earlier, experiments indicated that DP and DM dendrimers were efficiently complexing an anti TNF- $\alpha$ siRNA but complexes were shown more stable in vitro for DP, leading to more efficient anti-inflammatory effect by inhibition of TNF- $\alpha$ both in vitro and in vivo. Here, a coupled experimental/computational (ITC/Molecular Dynamics) approach was employed to shed light on mechanisms driving the dendrimer-siRNA complexation. Outcome of our research indicated DP to be more efficient in binding siRNA. In greater 
detail, DP higher efficiency with respect to DM lies in two main features: 1) DP-siRNA complexation maximizes the entropic contribution to the binding free energy and 2) DP requires a lower number of terminals to directly bind the siRNA while leaving a large part of them free to fluctuate under thermal motion to eventually catch another siRNA molecule.

Our work provides a further proof on the suitability of computational molecular modeling, such as Molecular Dynamics, coupled with high resolution experimental techniques, such as ITC. The suggested platform can be used $i$ ) to predict the efficiency of nanoparticle-siRNA hybrids to screen and select the best hits before further in vitro and in vivo tests, or ii) to deeply understand experimental results by providing fruitful insight in mechanism and molecular phenomena driving nanoparticle-siRNA complexation, as the case of DP and DM considered in this work.

\section{Conflicts of Interest}

There are no conflicts to declare

\section{Acknowledgements}

$A D$ and MAD gratefully acknowledge the financial support from the Swiss National Foundation through the grant IZCNZ0-174842 ("Computational modeling to characterize and optimize dendrimer based nanocarriers for siRNA delivery in Chronicle Obstructive Pulmonary Disease"). The authors would also like to acknowledge the financial support provided by COST-European Cooperation in Science and Technology, to the COST Action MP1404: Simulation and pharmaceutical technologies for advanced patient-tailored inhaled medicines (Siminhale). This work was also supported by the Swiss National Supercomputing Centre (CSCS).

\section{References}

1 J. C. Kaczmarek, P. S. Kowalski and D. G. Anderson, Genome Med., 2017, 9.

2 E. Fattal and G. Barratt, Br. J. Pharmacol., 2009, 157, 179-194.

3 R. D. Harvey, S. Bourgeois, P. Pietzonka, L. Desire and E. Fattal, Nanobiotechnology, 2005, 1, 071082.

$4 \quad$ W. Li and F. C. Szoka, Pharm. Res., 2007, 24, 438-49.

5 G. De Rosa, D. De Stefano, V. Laguardia, S. Arpicco, V. Simeon, R. Carnuccio and E. Fattal, Eur. J. Pharm. Biopharm. Off. J. Arbeitsgemeinschaft für Pharm. Verfahrenstechnik e.V, 2008, 70, 7-18. 
E. Fattal and A. Bochot, Int. J. Pharm., 2008, 364, 237-48.

F. Bruxel, S. Cojean, A. Bochot, H. Teixeira, C. Bories, P.-M. Loiseau and E. Fattal, Int. J. Pharm., 2011, 416, 402-9.

M. Fraga, F. Bruxel, V. L. Lagranha, H. F. Teixeira and U. Matte, Int. J. Nanomedicine, 2011, 6, 221320.

S. Nimesh, N. Gupta and R. Chandra, J. Biomed. Nanotechnol., 2011, 7, 504-20.

F. Bruxel, A. Bochot, D. Diel, L. Wild, E. L. S. Carvalho, S. Cojean, P. M. Loiseau, E. Fattal and H. F. Teixeira, Curr. Top. Med. Chem., 2014, 14, 1161-71.

H. Y. Xue, P. Guo, W.-C. Wen and H. L. Wong, Curr. Pharm. Des., 2015, 21, 3140-7.

M. J. Hope, B. Mui, S. Ansell and Q. F. Ahkong, Mol. Membr. Biol., 1998, 15, 1-14.

M. Zheng, G. M. Pavan, M. Neeb, A. K. Schaper, A. Danani, G. Klebe, O. M. Merkel and T. Kissel, ACS Nano, 2012, 6, 9447-54.

G. M. Pavan, P. Posocco, A. Tagliabue, M. Maly, A. Malek, A. Danani, E. Ragg, C. V Catapano and S. Pricl, Chemistry, 2010, 16, 7781-95.

N. Malik, R. Wiwattanapatapee, R. Klopsch, K. Lorenz, H. Frey, J. W. Weener, E. W. Meijer, W. Paulus and R. Duncan, J. Control. Release, 2000, 65, 133-48.

T. Barrett, G. Ravizzini, P. L. Choyke and H. Kobayashi, IEEE Eng. Med. Biol. Mag., 2009, 28, 12-22.

H. a. Bullen, J. Biomater. Nanobiotechnol., 2011, 2, 485-493.

C. DUfES, I. UCHEGBU and A. SCHATZLEIN, Adv. Drug Deliv. Rev., 2005, 57, 2177-2202.

S. Biswas and V. Torchilin, Pharmaceuticals, 2013, 6, 161-183.

D. Shcharbin, A. Shakhbazau and M. Bryszewska, Expert Opin. Drug Deliv., 2013, 10, 1687-98.

S. P. Chaplot and I. D. Rupenthal, J. Pharm. Pharmacol., 2014, 66, 542-56.

F. Aulenta, W. Hayes and S. Rannard, Eur. Polym. J., 2003, 39, 1741-1771.

S. Fruchon, M. Poupot, L. Martinet, C.-O. Turrin, J.-P. Majoral, J.-J. Fournié, A.-M. Caminade and R. Poupot, J. Leukoc. Biol., 2009, 85, 553-62.

M. Hayder, S. Fruchon, J.-J. Fournié, M. Poupot and R. Poupot, ScientificWorldJournal., 2011, 11, 1367-82.

K. Neibert, V. Gosein, A. Sharma, M. Khan, M. A. Whitehead, D. Maysinger and A. Kakkar, Mol. Pharm., 2013, 10, 2502-8.

J. Ledall, S. Fruchon, M. Garzoni, G. M. Pavan, A.-M. Caminade, C.-O. Turrin, M. Blanzat and R. Poupot, Nanoscale, 2015, 7, 17672-84.

O. Klementieva, N. Benseny-Cases, A. Gella, D. Appelhans, B. Voit and J. Cladera, Biomacromolecules, 2011, 12, 3903-9. 
E. Blattes, A. Vercellone, H. Eutamène, C.-O. Turrin, V. Théodorou, J.-P. Majoral, A.-M. Caminade, J. Prandi, J. Nigou and G. Puzo, Proc. Natl. Acad. Sci. U. S. A., 2013, 110, 8795-800.

G. M. Pavan, L. Albertazzi and A. Danani, J. Phys. Chem. B, 2010, 114, 2667-75.

G. M. Pavan, M. A. Mintzer, E. E. Simanek, O. M. Merkel, T. Kissel and A. Danani, Biomacromolecules, 2010, 11, 721-30.

G. M. Pavan and A. Danani, J. Drug Deliv. Sci. Technol., 2012, 22, 83-89.

G. M. Pavan and A. Danani, Curr. Drug Discov. Technol., 2011, 8, 314-328.

A. Bohr, N. Tsapis, I. Andreana, A. Chamarat, C. Foged, C. Delomenie, M. Noiray, N. El Brahmi, J.-P. Majoral, S. Mignani and E. Fattal, Biomacromolecules, 2017, 18, 2379-2388.

C. Sun, T. Tang and H. Uludağ, J. Phys. Chem. B, 2012, 116, 2405-2413.

G. M. Pavan, A. Danani, S. Pricl and D. K. Smith, J. Am. Chem. Soc., 2009, 131, 9686-94.

D. A. Tomalia, Soft Matter, 2010, 6, 456-474.

D. Shcharbin, V. Dzmitruk, A. Shakhbazau, N. Goncharova, I. Seviaryn, S. Kosmacheva, M. Potapnev, E. Pedziwiatr-Werbicka, M. Bryszewska, M. Talabaev, A. Chernov, V. Kulchitsky, A.-M. Caminade and J.-P. Majoral, Pharmaceutics, 2011, 3, 458-473.

M. Lepre, S. Omar, G. Grasso, U. Morbiducci, M. Deriu and J. Tuszynski, Molecules, 2017, 22, 1358.

A. Janaszewska, B. Klajnert-Maculewicz, M. Marcinkowska, P. Duchnowicz, D. Appelhans, G. Grasso, M. A. Deriu, A. Danani, M. Cangiotti and M. F. Ottaviani, Nano Res., 2018, 11, 1204-1226.

G. Grasso, M. A. Deriu, V. Patrulea, G. Borchard, M. Möller and A. Danani, PLoS One, 2017, 12, e0186816.

M. A. Deriu, L. M. Popescu, M. F. Ottaviani, A. Danani and R. M. Piticescu, J. Mater. Sci., 2016, 51, 1996-2007.

X. Feng, Y. Cheng, K. Yang, J. Zhang, Q. Wu and T. Xu, J. Phys. Chem. B, 2010, 114, 11017-11026.

C. V Kelly, M. G. Liroff, L. D. Triplett, P. R. Leroueil, D. G. Mullen, J. M. Wallace, S. Meshinchi, J. R. Baker, B. G. Orr and M. M. Banaszak Holl, ACS Nano, 2009, 3, 1886-96.

L. M. Popescu, R. M. Piticescu, G. Doni, A. Danani and L. M. Popescu, Piticescu R. M., Doni G., Danani A., Rev. Roum. Chim., 2012, 57, 35-38.

L. B. Jensen, G. M. Pavan, M. R. Kasimova, S. Rutherford, A. Danani, H. M. Nielsen and C. Foged, Int. J. Pharm., 2011, 416, 410-8.

M. A. Deriu, G. Grasso, G. Licandro, A. Danani, D. Gallo, J. A. Tuszynski and U. Morbiducci, PLoS One, 2014, 9, e108677.

G. Grasso, S. Muscat, M. Rebella, U. Morbiducci, A. Audenino, A. Danani and M. A. Deriu, J. Biomech., , DOI:10.1016/j.jbiomech.2018.03.036.

50 G. Grasso, U. Morbiducci, D. Massai, J. Tuszynki, A. Danani and M. Deriu, Biophys. J., 2018, 114, 323330. 
G. Grasso, M. Rebella, S. Muscat, U. Morbiducci, J. Tuszynski, A. Danani and M. Deriu, Int. J. Mol. Sci., $2018,19,571$.

52 M. D. Hanwell, D. E. Curtis, D. C. Lonie, T. Vandermeersch, E. Zurek and G. R. Hutchison, J. Cheminform., 2012, 4, 17.

J. Wang, R. M. Wolf, J. W. Caldwell, P. A. Kollman and D. A. Case, J. Comput. Chem., 2004, 25, 11571174.

A.-M. Caminade, S. Fruchon, C.-O. Turrin, M. Poupot, A. Ouali, A. Maraval, M. Garzoni, M. Maly, V. Furer, V. Kovalenko, J.-P. Majoral, G. M. Pavan and R. Poupot, Nat. Commun., 2015, 6, 7722.

J. Lim, G. M. Pavan, O. Annunziata and E. E. Simanek, J. Am. Chem. Soc., 2012, 134, 1942-5.

M. J. Abraham, T. Murtola, R. Schulz, S. Páll, J. C. Smith, B. Hess and E. Lindahl, SoftwareX, 2015, 1-2, $19-25$.

J. Wang, W. Wang, P. A. Kollman and D. A. Case, J. Comput. Chem., 2005, 25, 1157-1174.

J. Wang, W. Wang, P. A. Kollman and D. A. Case, J. Mol. Graph. Model., 2006, 25, 247-260.

A. W. Sousa da Silva and W. F. Vranken, BMC Res. Notes, 2012, 5, 367.

B. Hess, C. Kutzner, D. van der Spoel and E. Lindahl, J. Chem. Theory Comput., 2008, 4, 435-447.

V. Hornak, R. Abel, A. Okur, B. Strockbine, A. Roitberg and C. Simmerling, Proteins, 2006, 65, 712-25.

K. Lindorff-Larsen, S. Piana, K. Palmo, P. Maragakis, J. L. Klepeis, R. O. Dror and D. E. Shaw, Proteins, 2010, 78, 1950-8.

K. Lindorff-Larsen, P. Maragakis, S. Piana, M. P. Eastwood, R. O. Dror and D. E. Shaw, PLoS One, 2012, 7, e32131.

G. Bussi, D. Donadio and M. Parrinello, J. Chem. Phys., 2007, 126, 14101.

H. J. C. Berendsen, J. P. M. Postma, W. F. Van Gunsteren, A. DiNola and J. R. Haak, J. Chem. Phys., 1984, 81, 3684-3690.

B. Hess, H. Bekker, H. J. C. Berendsen and J. G. E. M. Fraaije, J. Comput. Chem., 1997, 18, 1463-1472.

W. Humphrey, A. Dalke and K. Schulten, J. Mol. Graph., 1996, 14, 33-8, 27-8.

T. Hou, J. Wang, Y. Li and W. Wang, J. Chem. Inf. Model., 2011, 51, 69-82.

S. Genheden and U. Ryde, Expert Opin. Drug Discov., 2015, 10, 449-461.

A. Martínez-Muñoz, M. Bello, A. Romero-Castro, R. A. Rodríguez-Fonseca, J. Rodrigues, V. A. Sánchez-Espinosa and J. Correa-Basurto, J. Mol. Graph. Model., 2017, 76, 330-341.

T. Hou, J. Wang, Y. Li and W. Wang, J. Comput. Chem., 2011, 32, 866-77.

A. T. Ayoub, M. Klobukowski and J. A. Tuszynski, PLoS Comput. Biol., 2015, 11, e1004313.

C. Wu, Z. Wang, H. Lei, W. Zhang and Y. Duan, J. Am. Chem. Soc., 2007, 129, 1225-32.

K. Bouchemal and S. Mazzaferro, Drug Discov. Today, 2012, 17, 623-629. 
77 V. Vasumathi and P. K. Maiti, Macromolecules, 2010, 43, 8264-8274. 